\title{
Magnetic imaging with femtosecond temporal resolution
}

\author{
Jie Li, ${ }^{1}$ Min-Sang Lee, ${ }^{1}$ Wei He, ${ }^{1}$ Björn Redeker, ${ }^{1}$ Arndt Remhof, ${ }^{1, a)}$ Edward Amaladass, ${ }^{2}$ \\ Christoph Hassel, ${ }^{3}$ and Thomas Eimüller ${ }^{1,4, b)}$ \\ ${ }^{1}$ Junior Research Group Magnetic Microscopy, Institute for Experimental Physics IV, \\ Ruhr-University of Bochum, Universitätsstr. 150, D-44780 Bochum, Germany \\ ${ }^{2}$ Max Planck Institute for Metals Research, Heisenbergstr. 3, D-70569 Stuttgart, Germany \\ ${ }^{3}$ Fachbereich Physik and Center for Nanointegration Duisburg-Essen (CeNIDE), Universität Duisburg-Essen, \\ Lotharstr. 1, D-47048 Duisburg, Germany \\ ${ }^{4}$ Hochschule Kempten, University of Applied Sciences, Bahnhofstr. 61, D-87435 Kempten, Germany
}

(Received 30 April 2009; accepted 15 June 2009; published online 14 July 2009)

A scanning Kerr microscope with a temporal resolution of $<230$ fs and a spatial resolution of $210 \mathrm{~nm}$ is presented. Equipped with a large temporal and spatial scanning range of $8 \mathrm{~ns}$ and $320 \mu \mathrm{m}$, respectively, the microscope allows studying nonuniform magnetization dynamics on many different time scales over a large area. For demonstration, we study the magnetization dynamics in $\mathrm{Fe} / \mathrm{Gd}$ multilayer dot arrays exhibiting a spin reorientation transition (SRT) on three different time scales, namely, femtosecond, picosecond, and nanosecond scales. The dynamics on all time scales varies from one dot to another. This is attributed to the high sensitivity of the SRT to the variations of the layer thicknesses and the Fe/Gd interface structure. (C) 2009 American Institute of Physics. [DOI: 10.1063/1.3170448]

\section{INTRODUCTION}

The objectives of modern data storage industry can be summarized by the slogan "smaller and faster." Over the last decade it fueled the efforts to develop novel concepts for high density storage media with rapid switching processes (see Refs. 1-4 and references therein). All these research activities are based on knowledge about the magnetization dynamics in magnetic structures on a nanometer scale. It is therefore crucial to develop experimental methods that can measure the dynamic response both with a high temporal and a high spatial resolution. Such imaging methods enable direct visualization of nonuniform spin precession, magnetostatic waves, and spin waves. ${ }^{5-10}$ In particular, these techniques are useful for studying local magnetization dynamics in magnetic dots, large arrays of which are deemed to be promising candidates for future applications.

Considering the spatial resolution, techniques using $\mathrm{x}$-ray magnetic circular dichroism (XMCD) such as magnetic transmission $\mathrm{x}$-ray microscopy ${ }^{11-13}$ and $\mathrm{x}$-ray photoemission electron microscopy (X-PEEM) $)^{7,14,15}$ appear to be the best choice for dynamic imaging. Due to the short wavelength, $\mathrm{x}$-ray imaging techniques have been shown to provide a spatial resolution down to $15 \mathrm{~nm}$ using microzone plates. ${ }^{16}$ However, in terms of temporal resolution, time-resolved Kerr microscopy using magneto-optic Kerr effect (MOKE) with visible light has a clear advantage over $\mathrm{x}$-ray imaging techniques. The femtosecond laser sources with pulse widths $<100$ fs enable the study of magnetization dynamics on a time scale below 1 ps (Refs. 17-22), whereas the electron

\footnotetext{
${ }^{a)}$ Present address: Empa, Swiss Federal Laboratories for Materials Testing and Research, Überlandstr. 129, CH-8600 Dübendorf, Switzerland.

${ }^{b)}$ Author to whom correspondence should be addressed. Electronic mail: thomas.eimueller@fh-kempten.de.
}

bunch length and the jitter limit the temporal resolution for x-ray techniques to about 30-100 ps. ${ }^{23}$ Although the recently developed "femtoslicing method" opened the door to subpicosecond magnetization dynamics for time-resolved XMCD measurements, ${ }^{23,24}$ the low flux does not allow combining this method with microscopy.

Therefore, at the current stage of technical development, imaging of magnetization dynamics in the femtosecond region is only possible by means of time-resolved scanning Kerr microscopy using the all-optical pump-and-probe technique. Several groups demonstrated such experimental setups with certain technical variations. In the setup developed by Freeman's group, ${ }^{25}$ a $500 \mathrm{~nm}$ focal spot is reached by a combination of a probe beam wavelength of $800 \mathrm{~nm}$ and an oil-immersion microscope objective with a high numerical aperture (NA) of 1.3. However, the used excitation of the sample by magnetic field pulses with a rise time of about $50 \mathrm{ps}$ limits the temporal resolution of this setup. This is also true for other setups applying magnetic field pulse excitation, such as the ones used by Back et $a l^{26}$ and Crowell and Compton. ${ }^{27}$ Recently it has been demonstrated by Münzenberg and co-workers that Auston switches made of lowtemperature grown GaAs generate field pulses with a rise time of few picoseconds. ${ }^{28}$

However, much shorter excitation is possible in all optical setups. Bigot and co-workers demonstrated a focal size of about $300 \mathrm{~nm}$ by focusing a $400 \mathrm{~nm}$ beam with an objective with NA $=0.65{ }^{22}$ Using a Ti:sapphire laser with a pulse duration of $180 \mathrm{fs}$, they reached a temporal resolution in the subpicosecond regime. Schmidt and co-workers ${ }^{29}$ did neither target on subpicosecond temporal resolution nor on imaging. However, they could measure the dynamics of nanomagnets with diameters as small as $125 \mathrm{~nm}$ by use of cavity enhancement of the MOKE. It is noteworthy that this technique en- 
ables studying structures that are much smaller $(\approx 8$ times) than the diameter of the used focal spot $(\approx 1 \mu \mathrm{m})$.

In this review we present our newly constructed femtosecond laser scanning Kerr microscope with a temporal resolution of $<230 \mathrm{fs}$ and a spatial resolution of $210 \mathrm{~nm}$, combined with a large temporal and spatial scanning range (8 ns and $320 \mu \mathrm{m}$, respectively). The performance and the functionalities of the setup are demonstrated by imaging the magnetization dynamics of a patterned $\mathrm{Co} / \mathrm{Pt}$ multilayer sample. To present in addition the ability to study lateral variations in the magnetization dynamics we carefully designed the layer structure of a Fe/Gd multilayer such that it exhibits a spin reorientation transition (SRT) at room temperature. Since the magnetic properties of this system are very sensitive even to small variations in the Fe/Gd bilayer thickness and the interface structure, ${ }^{30-32}$ this sample is expected to show local variations in the static and dynamic magnetic properties. We could confirm this by studying an unpatterned reference sample of a Fe/Gd multilayer using wide-field Kerr microscopy. A movie illustrating its magnetization reversal is provided as supplementary material downloadable from the Electronic Physics Auxiliary Publication Service (EPAPS) depository. ${ }^{33}$ It shows a remarkable reduction in the domain size over a distance of some microns, which clearly indicates a spatial variation in the magnetic properties.

\section{EXPERIMENTAL SETUP}

In our microscope a two-color all-optical pump-andprobe technique is implemented to study reproducible ultrafast magnetization dynamics. The optical setup of the microscope is shown in Fig. 1. Laser pulses with an output energy of ca. $80 \mathrm{~nJ}$ and a duration of ca. $55 \mathrm{fs}$ are obtained from a Ti:sapphire oscillator ("Scientific XL" from FEMTOLASERS) with a central wavelength of $780 \mathrm{~nm}$. The brevity, stability, and high output energy of the pulses are achieved with a long multipass cavity and a mirror-dispersion-control scheme using chirped mirrors. ${ }^{34}$

Pulses of the fundamental wavelength are used to pump the sample system. The low repetition rate of the laser of $11 \mathrm{MHz}$ gives the system sufficient time to relax back into the initial state before the next pump pulse arrives after about 91 ns. The response of the magnetic system to this excitation is probed after a variable delay time $\Delta t$ by pulses with a wavelength of $390 \mathrm{~nm}$, obtained by second-harmonic generation in a $100 \mu \mathrm{m}$ thick $\beta$ - $\mathrm{BaB}_{2} \mathrm{O}_{4}$ crystal. The optical path length of the pump beam is varied using a high precision linear stage with a travel range of $1.2 \mathrm{~m}$ and a positional resolution of $50 \mathrm{~nm}$. By folding the beam it allows varying the delay time $\Delta t$ within a range of $8 \mathrm{~ns}$ with a subfemtosecond resolution (see Fig. 1). Both beams are collinearly focused to the magnetic sample by a microscope objective (Zeiss EC Epiplan-Apochromat $100 \times / 0.95$ HD DIC), which has been specially selected by the manufacturer to conserve the polarization state of the transmitted light. For two-dimensional scan imaging and fine adjustment of the focus, we use a three-axis piezoelectric scanning stage

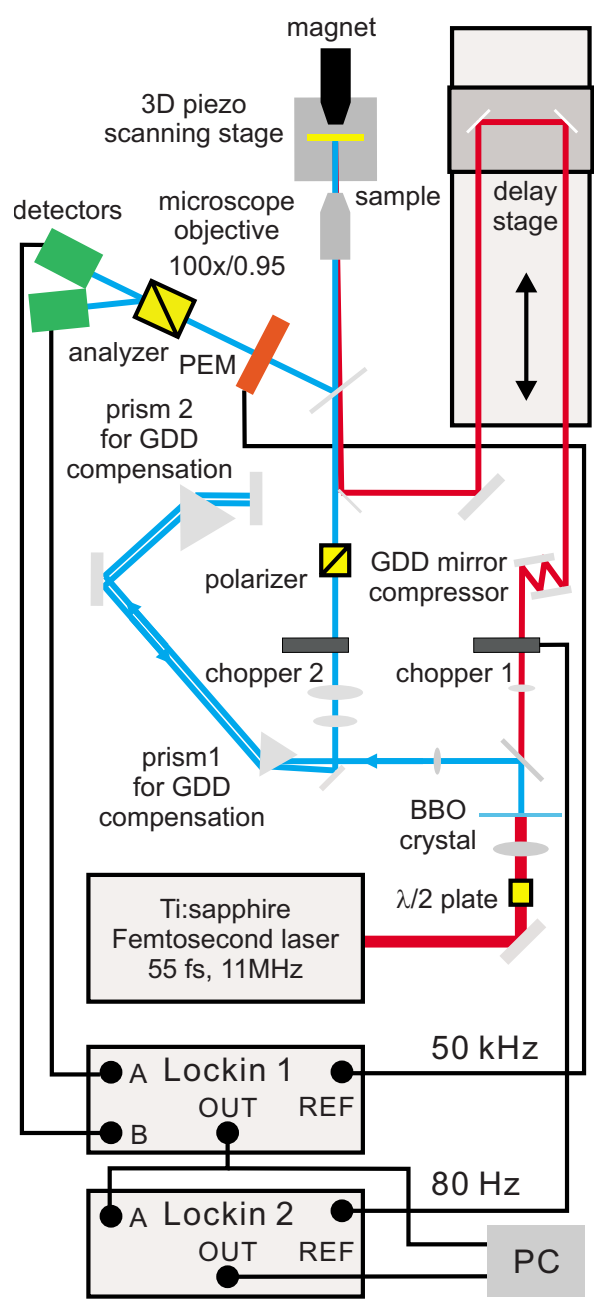

FIG. 1. (Color online) Schematic drawing of the optical setup of the femtosecond laser scanning Kerr microscope.

(TRITOR 400 CAP, nonmagnetic version, from piezosystem jena, Inc.) with a travel range of $320 \mu \mathrm{m}$ and a positioning resolution of $1 \mathrm{~nm}$ in all three axes.

The temporal resolution is limited by the duration of the probe pulses. As they propagate through many optical components, such as lenses, prisms, and, in particular, the microscope objective, the pulse duration is increased by groupdelay dispersion (GDD). To reduce this effect, all optical components have been selected for a minimal GDD. In addition, we induce negative GDD for compensation, using two prisms for the probe beam and a pair of chirped mirrors for the pump beam (see Fig. 1). ${ }^{35}$

To enhance the signal-to-noise ratio a double modulation technique is applied. ${ }^{36}$ For the detection of the static MOKE signal the probe beam is $p$-polarized by a Glan-air prism (extinction ratio of $10^{-6}$ ) and its polarization is modulated after the reflection from the sample by a photoelastic modulator (PEM) at a frequency of $50 \mathrm{kHz}$. After passing through a Rochon prism analyzer (extinction ratio of $10^{-6}$ ) the beam is detected by a GaP photodiode. The voltage output of the detector contains three types of signals at different frequencies: (i) dc voltage, proportional to the reflectivity, (ii) the first harmonic signal at the modulation frequency, proportional to the Kerr ellipticity $\epsilon_{K}$, (iii) the second harmonic 
signal at $100 \mathrm{kHz}$, proportional to the Kerr rotation $\theta_{K}$. A lock-in amplifier extracts the Kerr ellipticity and Kerr rotation signal at the given frequencies, while filtering out the noise at other frequencies. The pump-beam-induced changes in the Kerr ellipticity, $\Delta \epsilon_{K}$, and the Kerr rotation, $\Delta \theta_{K}$, reflect the dynamic response of the magnetic system. The signal difference between the perturbed and unperturbed state is realized by chopping the pump beam at a frequency of 80 Hz. At this frequency another lock-in amplifier extracts $\Delta \epsilon_{K}$ and $\Delta \theta_{K}$ from the output of the first lock-in. This two level noise filtering procedure using double lock-in detection, first introduced by Koopmans et al. ${ }^{36}$ in nonimaging dynamic MOKE experiments, gives a great improvement of the signal-to-noise ratio. Many dynamic imaging experiments use a balanced detector to detect the small differences in the intensity of the $s$ - and $p$-polarized beams that result from changes in the Kerr rotation. ${ }^{37,38}$ In contrast, our approach using a PEM gives a higher sensitivity and enables simultaneous measuring of both Kerr rotation and Kerr ellipticity.

All measurement data that will be presented below were obtained in the polar MOKE geometry making the experimental setup sensitive only to the magnetization component perpendicular to the sample plane. The simultaneous measurement of the other components is also possible using a quadrant detector, ${ }^{39}$ but this option has not been implemented for the present work. Time-resolved reflectivity measurements are also performed using the double lock-in technique. For this purpose another chopper (chopper 2 in Fig. 1) is used instead of the PEM to modulate the probe beam intensity at $2 \mathrm{kHz}$. An out-of-plane magnetic field of up to $\pm 300 \mathrm{mT}$ can be applied to the sample using an electromagnet with a bipolar operational power supply. A controlling software for the whole system is developed based on the Microsoft Visual Studio platform, which allows us to drive and read out all the electric and electronic components of the microscope. It enables scan procedures where arbitrary combinations of parameters, namely, the three spatial axes, the delay time, and the external magnetic field, can be selected. Along these scan axes the corresponding signals, i.e, the transient reflectivity $\Delta R$, the Kerr rotation $\theta_{K}$ and ellipticity $\epsilon_{K}$, and the dynamic Kerr rotation $\Delta \theta_{K}$ and ellipticity $\Delta \epsilon_{K}$ can be recorded. This gives us a great flexibility to perform experiments such as two-dimensional mapping of local hysteresis loops, measurement of hysteresis loops at different delay times, two-dimensional imaging of the dynamic optic, magneto-optic response of the sample at different delay times, etc.

\section{RESULTS AND DISCUSSION}

To determine the temporal and spatial resolution of the microscope, we have first carried out test measurements. The temporal resolution of the microscope is estimated by measuring the ultrafast demagnetization process in a Fe/Gd multilayer $([\mathrm{Fe}(0.4 \mathrm{~nm}) / \mathrm{Gd}(0.4 \mathrm{~nm})] \times 70)$, deposited on a $\mathrm{Si}$ substrate by $\mathrm{rf}$ ion sputtering and capped with a $2 \mathrm{~nm}$ thick $\mathrm{Al}$ layer for protection against oxidation. The thickness of the Fe and Gd layers was tuned close to the SRT to enable optothermic excitation of a spin precession. ${ }^{7}$ By optimizing
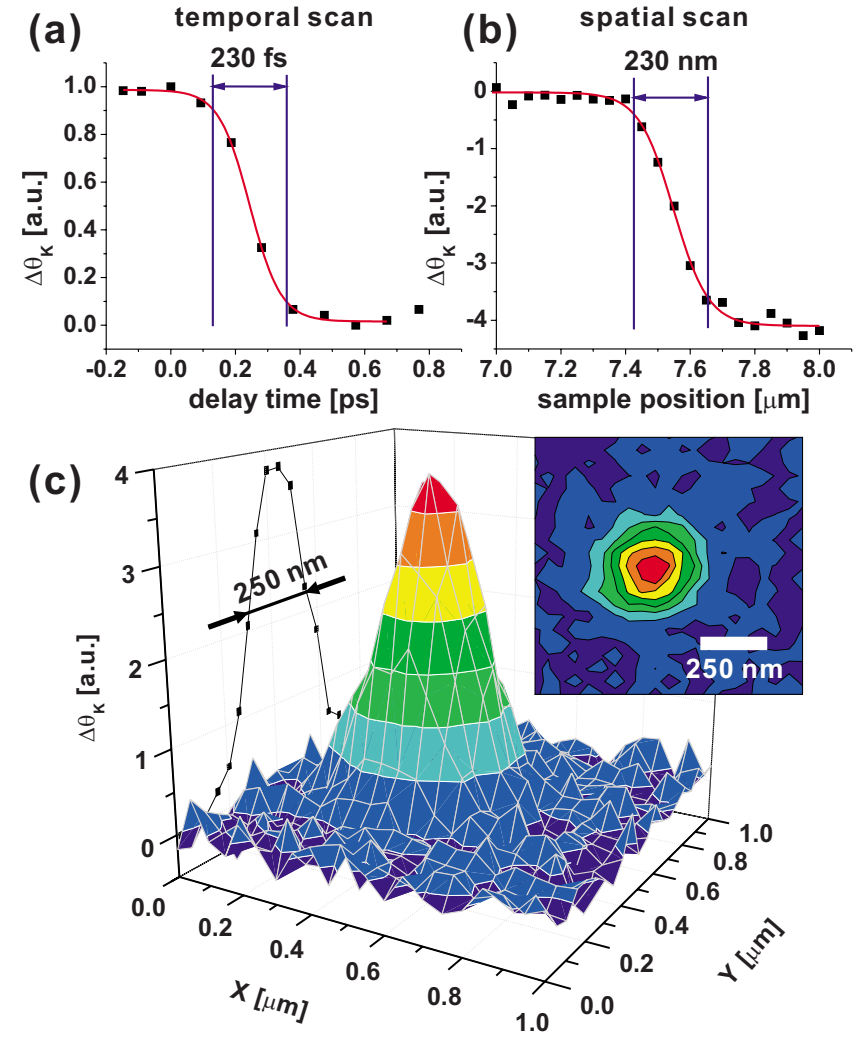

FIG. 2. (Color online) Resolution tests. (a) The dynamic Kerr rotation signal $\Delta \theta_{K}$ of a $\mathrm{Fe} / \mathrm{Gd}$ multilayer film recorded during the demagnetization process; the signal decreases within 230 fs from $90 \%$ to $10 \%$ of the signal contrast. (b) A spatial line scan of a sharp edge on a structured $\mathrm{Co} / \mathrm{Pt}$ multilayer sample revealing a width of $230 \mathrm{~nm}$ between $90 \%$ and $10 \%$ of the signal contrast. (c) The spatial and temporal resolution is combined to record a two-dimensional image of the dynamic Kerr response from a $\mathrm{Co} / \mathrm{Pt}$ dot with a diameter of $200 \mathrm{~nm}$ at the delay time of the maximum demagnetization.

the setting for the aforementioned compressor mirrors and prisms, we reduced the GDD for both the pump and the probe beam until a minimum value of $230 \mathrm{fs}$ was reached for the demagnetization time, as shown in Fig. 2(a). Note that this value is an upper limit for the temporal resolution. Assuming that the $\mathrm{Fe} / \mathrm{Gd}$ sample responds to the pump pulse within $150 \mathrm{fs}$, a temporal resolution below $200 \mathrm{fs}$ is obtained. For this estimation the convolution of a Gaussian beam profile with an exponential response of the sample has been used to reproduce the fitting curve to the experimental data shown in Fig. 2(a). ${ }^{40}$

For the estimation of the spatial resolution, we use a structured $\mathrm{Co} / \mathrm{Pt}$ multilayer $([\mathrm{Co}(0.25 \mathrm{~nm}) / \mathrm{Pt}(0.95 \mathrm{~nm})]$ $\times 20)$ sample with a $1 \mathrm{~nm}$ thick Pt capping layer, fabricated in an e-beam evaporation system and structured by e-beam lithography. This system was designed to exhibit very strong perpendicular magnetic anisotropy. ${ }^{41}$ The delay time $\Delta t$ is set to the point of the maximum demagnetization and the dynamic Kerr rotation signal $\Delta \theta_{K}$ is recorded while scanning over a sharp edge of the structured $\mathrm{Co} / \mathrm{Pt}$ multilayer sample, see Fig. 2(b). Again, the measured curve represents the convolution between the Gaussian point spread function of the focused laser spot and the topographic profile of the step (Heavyside function). Fitting this convolution reveals a spatial resolution of ca. $210 \mathrm{~nm}$ (full width at half maximum of 


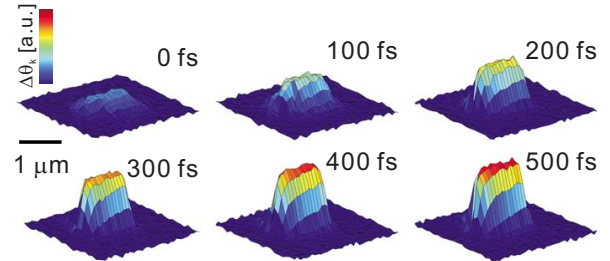

FIG. 3. (Color online) The ultrafast demagnetization in an elliptical $\mathrm{Co} / \mathrm{Pt}$ multilayer dot with a semimajor axis length of $2 \mu \mathrm{m}$ and a semiminor axis length of $1 \mu \mathrm{m}$.

the Gaussian profile), which is between the diffraction limits defined by the Rayleigh resolution criterion (0.61 $\lambda / \mathrm{NA}$, where $\lambda=390 \mathrm{~nm}$ is the wavelength of the light and NA $=0.95$ is the numerical aperture of the microscope objective) and the Sparrow criterion (0.47 $\lambda / \mathrm{NA})$. Adding another axis to the spatial scan, a two-dimensional image of the maximum demagnetization of a $\mathrm{Co} / \mathrm{Pt}$ multilayer nanodot with a diameter of $200 \mathrm{~nm}$ could be recorded [see Fig. 2(c)].

Combining two-dimensional spatial imaging with temporal scanning, one can produce a stack of dynamic MOKE images at different delay times as illustrated in Fig. 3. Here, the ultrafast demagnetization process in a micron-sized $\mathrm{Co} / \mathrm{Pt}$ dot is spatiotemporally resolved. The spatial distribution of the dynamic MOKE signal $\Delta \theta_{K}$ inside the dot indicates that the demagnetization process occurs quite homogeneously within the given spatial resolution and signal sensitivity.

The ability to perform time-resolved Kerr microscopy with such high spatiotemporal resolution and large scanning ranges is very useful, in particular, if samples that exhibit different local magnetization dynamics at different positions need to be studied. For demonstration, we recorded dynamic MOKE images for 25 different Fe/Gd multilayer dots with a diameter of $2 \mu \mathrm{m}$ at 46 different delay times, as shown in Fig. 4. Before the experiments, a perpendicular external field strong enough to saturate the sample magnetization was applied using an electromagnet and then switched off so that the sample was in a remanent state during the scanning processes.

From the sequence of the dynamic MOKE image we created a movie, which is available in the EPAPS depository. ${ }^{33}$ It shows that the dynamics differs from one dot to another on three time scales: (1) the ultrafast demagnetization in the subpicosecond range, (2) a fast oscillation in the subnanosecond range, and (3) the slow spin precession caused by the heat induced SRT on the nanosecond scale. For the sake of clarity, we selected only four dots out of the 25 for discussion and show their behavior in Fig. 4, where two of them (C and D) exhibit qualitatively the same dynamics and the other two (A and B) distinctively different dynamics. The slow spin precession caused by the heat-induced SRT was already studied in more detail by Eimüller et al. using X-PEEM, ${ }^{7}$ however, due to the limited temporal resolution of the synchrotron method they were not able to resolve the other dynamics occurring on shorter time scales.

An ultrafast demagnetization is observed in all four dots at the same delay time. However, there is a clear difference between the dots in the extent of their demagnetization. The negative demagnetization peaks of $\operatorname{dot} \mathrm{A}$ and $\mathrm{B}$ are much

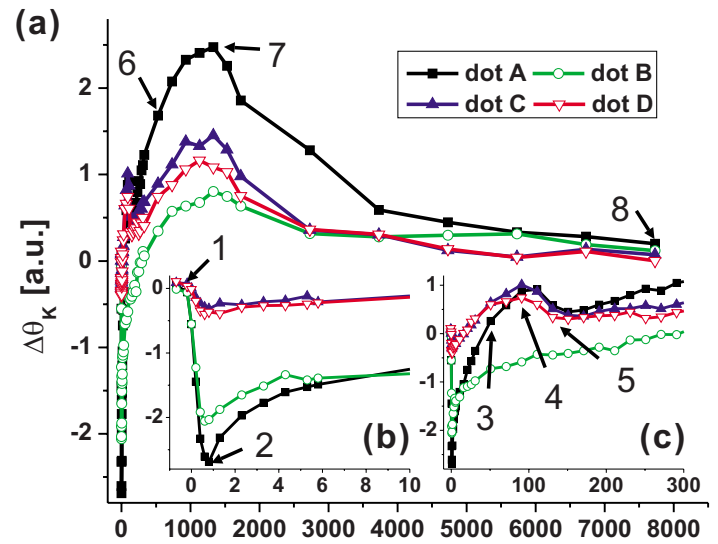

(d)

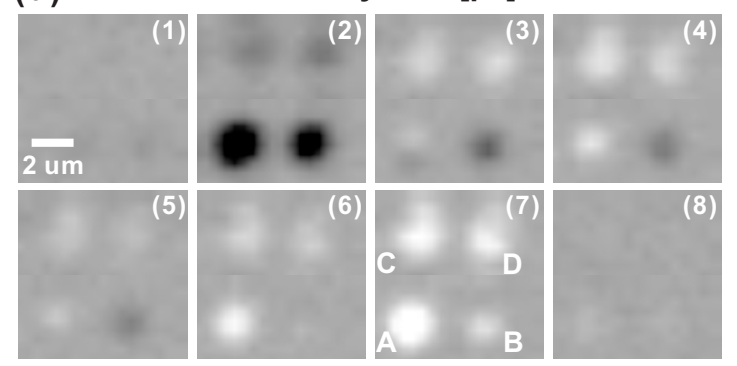

FIG. 4. (Color online) The magnetization dynamics in an Fe/Gd multilayer dot array: (a) the dynamic MOKE signal $\Delta \theta_{K}$ of four dots, marked with A-D in the dynamics MOKE image (7) below, within the $8 \mathrm{~ns}$ range; position (7) marks the first peak of the SRT-induced spin precession. (b) and (c) resolve the ultrafast demagnetization and a perpendicular standing spin wave during the remagnetization, respectively. (d) The dynamic MOKE images (1)-(8) of the four dots recorded at eight different delay times denoted by 1-8 in the graph for dot A within (a).

more pronounced than those of $\operatorname{dot} \mathrm{C}$ and $\mathrm{D}$; compare the dynamic MOKE image (2) in Fig. 4(d) and the curves in the temporally magnified inset of Fig. 4(b). The differences in dynamics are not only observed in this subpicosecond range, but also during the remagnetization process. First, the dots recover their magnetization with different speeds. Second, during the recovery in the subnanosecond range, a strongly damped fast oscillation appears for some dots [see inset of Fig. 4(c) and images (3-5) of Fig. 4(d)] and was identified as a perpendicular standing spin wave. However, there are also dots that do not show this oscillation at all, e.g., dot B. In addition, the oscillation amplitudes and their decay are different in general from one dot to another. The oscillation period, however, is the same for all dots and has a value of ca. 120 ps. Third, a large increase in the dynamic MOKE signal, which is attributed to the heat-induced SRT, is observed in Fig. 4(a) at a delay time $\Delta t \approx 1 \mathrm{~ns}$ and, also here, the height of the peak is different from one dot to another, but the peak appears at the same delay time for all dots; also compare the dynamic MOKE image (7) in Fig. 4(d). A possible explanation of these differences is local variations in the structure of the Fe/Gd multilayer. According to previous studies on thin Fe/Gd multilayer systems, ${ }^{31,32}$ the intrinsic magnetic anisotropy and the magnetic moments strongly depend on the thickness of the single Fe/Gd bilayers, the composition, and the structure of intermetallic compounds at the Fe/Gd interfaces. The sample area on which the studied dots were fabricated may contain defects in the structure and/or 
small positional variations in structural properties, such as inhomogeneities of the thickness and interfacial roughness. Therefore, dots with such slight variations in the multilayer structure will exhibit different magnetization dynamics. The results from detailed studies on the magnetization dynamics in Fe/Gd multilayers will be published elsewhere.

\section{SUMMARY AND CONCLUSION}

To summarize, we presented a valuable tool for the investigation of the local magnetization dynamics in ferromagnetic nanoelements. High temporal and spatial resolution $(<230$ fs and $210 \mathrm{~nm}$, respectively) are combined with large scanning ranges (8 ns and $320 \mu \mathrm{m})$ and a high signal-tonoise ratio in one setup. This allowed us to observe the magnetization dynamics on three different time scales and their spatial variation of $\mathrm{Fe} / \mathrm{Gd}$ multilayer systems. Using oilimmersion objectives with high numerical apertures such as $\mathrm{NA}=1.4$, the spatial resolution can be further improved. By doing so, we are currently studying local magnetization dynamics on smaller length scales $(\approx 100 \mathrm{~nm})$. We are also trying to improve the temporal resolution by using a reflective objective thus minimizing the GDD. Such attempts to push the temporal and spatial resolution of time-resolved magnetic imaging techniques to their limits will provide deeper insights into interesting physics such as the microscopic mechanisms and interactions responsible for different types of magnetization dynamics. In addition, this will ultimately lead to the development of novel devices fulfilling the demands made by modern data storage industry with the slogan "smaller and faster."

\section{ACKNOWLEDGMENTS}

We thank Professor H. Zabel, Dr. J. Lindner, and Professor G. Schütz for the permission to use the equipments in their institutes for sample fabrication. We also gratefully acknowledge the financial support by the DFG provided in the framework of the SFB 491 (project N1).

${ }^{1}$ J. Fidler, T. Schrefl, V. D. Tsiantos, W. Scholz, D. Suess, and H. Forster, J. Appl. Phys. 91, 7974 (2002).

${ }^{2}$ S. Garzon, L. Ye, R. A. Webb, T. M. Crawford, M. Covington, and S. Kaka, Phys. Rev. B 78, 180401 (2008), and references therein.

${ }^{3}$ S.-K. Kim, K.-S. Lee, Y.-S. Yu, and Y.-S. Choi, Appl. Phys. Lett. 92, 022509 (2008).

${ }^{4}$ C. D. Stanciu, F. Hansteen, A. V. Kimel, A. Kirilyuk, A. Tsukamoto, A. Itoh, and Th. Rasing, Phys. Rev. Lett. 99, 047601 (2007), and references therein.

${ }^{5}$ Y. Acremann, C. H. Back, M. Buess, O. Portmann, A. Vaterlaus, D. Pescia, and H. Melchior, Science 290, 492 (2000).

${ }^{6}$ M. Buess, R. Höllinger, T. Haug, K. Perzlmaier, U. Krey, D. Pescia, M. R. Scheinfein, D. Weiss, and C. H. Back, Phys. Rev. Lett. 93, 077207 (2004).

${ }^{7}$ T. Eimüller, A. Scholl, B. Ludescher, G. Schütz, and J.-U. Thiele, Appl. Phys. Lett. 91, 042508 (2007).

${ }^{8}$ F. Wegelin, A. Krasyuk, H.-J. Elmers, S. A. Nepijko, C. M. Schneider, and G. Schönhense, Surf. Sci. 601, 4694 (2007).

${ }^{9}$ J. P. Park, P. Eames, D. M. Engebretson, J. Berezovsky, and P. A. Crowell, Phys. Rev. Lett. 89, 277201 (2002).

${ }^{10}$ V. V. Kruglyak, P. S. Keatley, A. Neudert, M. Delchini, and R. J. Hicken, Phys. Rev. B 77, 172407 (2008).

${ }^{11}$ H. Stoll, A. Puzic, B. V. Waeyenberge, P. Fischer, J. Raabe, M. Buess, T.
Haug, R. Höllinger, C. H. Back, D. Weiss, and G. Denbeau, Appl. Phys. Lett. 84, 3328 (2004).

${ }^{12}$ A. Puzic, B. Van Waeyenberge, K. W. Chou, P. Fischer, H. Stoll, G. Schütz, T. Tyliszczak, K. Rott, H. Brückl, G. Reiss, I. Neudecker, T. Haug, M. Buess, and C. H. Back, J. Appl. Phys. 97, 10E704 (2005).

${ }^{13}$ B. Van Waeyenberge, A. Puzic, H. Stoll, K. W. Chou, T. Tyliszczak, R. Hertel, M. Fähnle, H. Brückl, K. Rott, G. Reiss, I. Neudecker, D. Weiss, C. H. Back, and G. Schütz, Nature (London) 444, 461 (2006).

${ }^{14}$ S.-B. Choe, Y. Acremann, A. Scholl, A. Bauer, A. Doran, J. Stöhr, and H. A. Padmore, Science 304, 420 (2004).

${ }^{15}$ J. Raabe, C. Quitmann, C. H. Back, F. Nolting, S. Johnson, and C. Buehler, Phys. Rev. Lett. 94, 217204 (2005).

${ }^{16}$ W. Chao, B. H. Harteneck, J. A. Liddle, E. H. Anderson, and D. T. Attwood, Nature (London) 435, 1210 (2005); D.-H. Kim, P. Fischer, W. Chao, E. Anderson, M.-Y. Im, S.-C. Shin, and S.-B. Choe, J. Appl. Phys. 99, $08 \mathrm{H} 303$ (2006).

${ }^{17}$ E. Beaurepaire, J.-C. Merle, A. Daunois, and J.-Y. Bigot, Phys. Rev. Lett. 76, 4250 (1996)

${ }^{18}$ J. Hohlfeld, E. Matthias, R. Knorren, and K. H. Bennemann, Phys. Rev. Lett. 78, 4861 (1997).

${ }^{19}$ B. Koopmans, M. van Kampen, J. T. Kohlhepp, and W. J. M. de Jonge, Phys. Rev. Lett. 85, 844 (2000).

${ }^{20}$ H. S. Rhie, H. A. Dürr, and W. Eberhardt, Phys. Rev. Lett. 90, 247201 (2003).

${ }^{21}$ J.-Y. Bigot, L. Guidoni, E. Beaurepaire, and P. N. Saeta, Phys. Rev. Lett. 93, 077401 (2004).

${ }^{22}$ A. Laraoui, M. Albrecht, and J.-Y. Bigot, Opt. Lett. 32, 936 (2007); A. Laraoui, J. Vénuat, V. Halté, M. Albrecht, E. Beaurepaire, and J.-Y. Bigot, J. Appl. Phys. 101, 09C105 (2007).

${ }^{23}$ S. Khan, K. Holldack, T. Kachel, R. Mitzner, and T. Quast, Phys. Rev. Lett. 97, 074801 (2006).

${ }^{24}$ C. Stamm, T. Kachel, N. Pontius, R. Mitzner, T. Quast, K. Holldack, S. Khan, C. Lupulescu, E. F. Aziz, M. Wietstruk, H. A. Dürr, and W. Eberhardt, Nature Mater. 6, 740 (2007).

${ }^{25}$ W. K. Hiebert, G. E. Ballentine, L. Lagae, R. W. Hunt, and M. R. Freeman, J. Appl. Phys. 92, 392 (2002).

${ }^{26}$ K. Perzlmaier, G. Woltersdorf, and C. H. Back, Phys. Rev. B 77, 054425 (2008).

${ }^{27}$ R. L. Compton and P. A. Crowell, Phys. Rev. Lett. 97, 137202 (2006).

${ }^{28}$ Z. Wang, M. Pietz, J. Walowski, A. Forster, M. I. Lepsa, and M. Münzenberg, J. Appl. Phys. 103, 123905 (2008).

${ }^{29}$ A. Barman, S. Wang, J. D. Maas, A. R. Hawkins, S. Kwon, A. Liddle, J. Bokor, and H. Schmidt, Nano Lett. 6, 2939 (2006).

${ }^{30}$ T. Kato, Y. Fujiwara, K. Yano, S. Tsunashima, S. Iwata, and M. Masuda, IEEE Trans. Magn. 36, 2909 (2000).

${ }^{31}$ E. Stavrou, H. Rohrmann, and K. Röll, IEEE Trans. Magn. 34, 1988 (1998); E. Stavrou and K. Röll, J. Appl. Phys. 85, 5971 (1999).

${ }^{32}$ T. Eimüller, E. Amaladass, and B. Ludescher (unpublished).

${ }^{33}$ See EPAPS supplementary material at http://dx.doi.org/10.1063/ 1.3170448 for the QUICKTIME movie "Supplementary_Material.mov" showing (top) the magnetization reversal process of an unpatterned $\mathrm{Fe} / \mathrm{Gd}$ multilayer film with structural inhomogeneities and (bottom) the optothermally induced magnetization dynamics in $25 \mathrm{Fe} / \mathrm{Gd}$ multilayer dots with a diameter of 2 micrometers.

${ }^{34}$ A. Fuerbach, A. Fernandez, A. Apolonski, E. Seres, T. Fuji, and F. Krausz, Proc. SPIE 5340, 4 (2004).

${ }^{35}$ R. Szipöcs, K. Ferencz, C. Spielmann, and F. Krausz, Opt. Lett. 19, 201 (1994).

${ }^{36}$ B. Koopmans, M. van Kampen, J. T. Kohlhepp, and W. J. M. de Jonge, J. Appl. Phys. 87, 5070 (2000).

${ }^{37}$ I. Neudecker, K. Perzlmaier, F. Hoffmann, G. Woltersdorf, M. Buess, D. Weiss, and C. H. Back, Phys. Rev. B 73, 134426 (2006).

${ }^{38}$ T. Kampfrath, R. G. Ulbrich, F. Leuenberger, M. Münzenberg, B. Sass, and W. Felsch, Phys. Rev. B 65, 104429 (2002).

${ }^{39}$ W. W. Clegg, N. A. E. Heyes, E. W. Hill, and C. D. Wright, J. Magn. Magn. Mater. 95, 49 (1991); T. J. Silva and A. B. Kos, J. Appl. Phys. 81, 5015 (1997)

${ }^{40}$ E. Carpene, E. Mancini, C. Dallera, M. Brenna, E. Puppin, and S. de Silvestri, Phys. Rev. B 78, 174422 (2008).

${ }^{41}$ V. W. Guo, B. Lu, X. Wu, G. Ju, B. Valcu, and D. Weller, J. Appl. Phys. 99, 08E918 (2006). 